\title{
The association between early MRI and length of disability in acute lower back pain: a systematic review and narrative synthesis
}

\author{
Bara A. Shraim', Muath A. Shraim², Ayman R. Ibrahim', Mohamed E. Elgamal', Basem Al-Omari, ${ }^{3,4}$ and \\ Mujahed Shraim ${ }^{5^{*}}$
}

\begin{abstract}
Background: Clinical guideline recommendations are against early magnetic resonance imaging (eMRI) within the first 4 to 6 weeks of conservative management of acute low back pain (LBP) without "clinical suspicion" of serious underlying conditions (red flags). There is some limited evidence that a significant proportion of patients with LBP receive eMRI non- indicated by clinical guidelines, which could be associated with increased length of disability (LOD). The aim of this systematic review was to investigate whether eMRI for acute LBP without red flags is associated with increased LOD. The LOD was defined as the number of disability days (absence from work).
\end{abstract}

Methods: Medline, EMBASE, and CINAHL bibliographic databases were searched from inception until June 5, 2021. Two reviewers independently assessed the methodological quality of included studies using the Newcastle-Ottawa scale and extracted data for the review. The search identified 324 records, in which seven studies met the inclusion criteria. Three of the included studies used the same study population. Owing to between-study heterogeneity, a narrative synthesis of results was used.

Results: All included studies were of good methodological quality and consistently reported that patients with acute LBP without red flags who received eMRI had increased LOD compared to those who did not receive eMRI. Three retrospective cohort studies reported that the eMRI groups had a higher mean LOD than the no eMRI groups ranging from 9.4 days $(95 \% \mathrm{Cl} 8.5,10.2)$ to 13.7 days $(95 \% \mathrm{Cl} 13.0,14.5)$ at the end of 1 -year follow-up period. The remaining studies reported that the eMRI groups had a higher hazard ratio of work disability ranging between $1.75(95 \% \mathrm{Cl} 1.23$, 2.50) and $3.57(95 \% \mathrm{Cl} 2.33,5.56)$ as compared to the no eMRI groups.

Conclusion: $\mathrm{eMRI}$ is associated with increased LOD in patients with acute LBP without red flags. Identifying reasons for performing non-indicated eMRI and addressing them with quality improvement interventions may improve adherence to clinical guidelines and improve disability outcomes among patients with LBP.

Keywords: Systematic review, Magnetic resonance imaging, Low back pain, Sick leave, Work disability, Return to work

*Correspondence: mshraim@qu.edu.qa

${ }^{5}$ Department of Public Health, College of Health Sciences, Qatar University, QU Health, Doha, Qatar

Full list of author information is available at the end of the article

\section{Background}

Low back pain (LBP) is ranked first globally for years lived with disability among all diseases with an estimated age-standardized point prevalence in 2017 at $7.5 \%$ [1]. Further, costs of managing for LBP are very high, exceeding $\$ 100$ billion per year in the US, and are still increasing $[2,3]$. A wide range of complex inter-related original author(s) and the source, provide a link to the Creative Commons licence, and indicate if changes were made. The images or other third party material in this article are included in the article's Creative Commons licence, unless indicated otherwise in a credit line to the material. If material is not included in the article's Creative Commons licence and your intended use is not permitted by statutory regulation or exceeds the permitted use, you will need to obtain permission directly from the copyright holder. To view a copy of this licence, visit http://creativecommons.org/licenses/by/4.0/. The Creative Commons Public Domain Dedication waiver (http://creativeco mmons.org/publicdomain/zero/1.0/) applies to the data made available in this article, unless otherwise stated in a credit line to the data. 
factors are associated with an increased length of disability (LOD) among individuals presenting with acute LBP. These include individual factors (e.g., age and gender) [4], occupational factors (e.g., job tenure, physical demand of job, workplace support) [5], regional factors (e.g., workers' compensation policies [6], socioeconomic factors [7]), and healthcare-related factors (e.g., early opioid prescribing within 15 days of LBP onset [8], early magnetic resonance imaging (eMRI) within the first 4-6 weeks of LBP onset) $[9,10]$. In this review, LOD was defined as the number of disability days (absence from work) due to the current episode of LBP [7, 11-13].

It is commonly observed that MRI findings of agerelated degenerative changes are prevalent in people without LBP $[14,15]$. In addition, a recent study found no relationship between MRI changes in the lumbar spine and pain intensity, health-related quality of life, and depressive and anxiety symptoms among patients with LBP [16]. Furthermore, a systematic review and metaanalysis of imaging strategies for LBP showed that lumbar imaging does not improve clinical outcomes in acute LBP cases without suspected serious underlying conditions [17].

Clinical guidelines for the management of acute nonspecific LBP recommend that imaging, specifically MRI, should not be performed in the first month of conservative management unless red flags (e.g., fracture, tumor, infection, and neurological deficit) are suspected [18-21]. Despite this, eMRI scanning for patients with acute LBP is common (27.7\%; $95 \%$ confidence interval (CI) $21.3,35.1$ ) [22] and was found to be associated with increased LOD, more healthcare utilization, and higher medical costs [10-12]. For instance, Mahmud et al., found that eMRI was associated with increased LOD by 102 days (unadjusted 115 vs. 13 days in eMRI and no eMRI groups, respectively) [12], whereas Graves et al., reported that eMRI was associated with an unadjusted 120-day increase in LOD [10]. Undertaking eMRI has been hypothesized to lead healthcare providers to overinterpret the findings and carry out additional and possibly unnecessary interventions, such as surgery, epidural steroid injections physiotherapy, osteopathy, and hospital admission [23-25] and thus lead to an increased LOD [12].

With multiple studies showing an independent association between eMRI and increased LOD among patients with acute LBP, it becomes necessary to synthesize the evidence from those studies. To our knowledge, only one systematic review has assessed the relationship between imaging, including MRI, and absence from work in patients with acute LBP [25]. However, that systematic review did not employ a specific timing for MRI scanning for LBP and included only two studies examining the relationship between eMRI and LOD in LBP cases and synthesized the findings using unadjusted LOD estimates between the eMRI and MRI groups. We are aware of more than two studies reporting on this relationship. Therefore, the aim of this systematic review is to summarize the findings of epidemiologic studies examining the relationship between eMRI and LOD in patients with acute LBP without "clinical suspicion" of serious underlying conditions (hereafter referred to as red flags).

\section{Materials and methods \\ Search strategy}

The protocol for this review was registered with the International Prospective Register of Systematic Reviews (PROSPERO) under registration number CRD42021259296 (available from https://www.crd.york. ac.uk/prospero/display_record.php? RecordID $=259,296$ ). Reporting of this systematic review was guided by the Preferred Reporting Items for Systematic Reviews and Meta-Analysis (PRISMA) statement (Supplemental file S1) [26]. We searched Medline, EMBASE, and CINAHL bibliographic databases from their inception until June 5, 2021, using medical subject heading (MeSH) or Emtree and free-text terms on LBP, MRI, and work disability (Supplemental file S2). In addition, reference lists of all relevant papers were searched, and citations of included studies were tracked using the Web of Science Citation Index. No restrictions on language, study design, or time of publication were applied.

\section{Criteria for considering studies for the review Types of studies}

All epidemiologic study designs examining the association between eMRI and LOD in patients with acute LBP were considered for inclusion.

\section{Types of participants}

Patients with a medical diagnosis of acute LBP, occupational LBP or non-specific LBP were included. Studies including patients with chronic or complicated LBP (e.g., severe injuries, multiple traumas, infection, autoimmune disease, or cancer) were not considered for inclusion in the review.

\section{Types of exposures}

The exposure was eMRI defined as an MRI of the lumbar spine for LBP within the first 4 to 6 weeks of the first recorded medical visit for the current LBP episode.

\section{Types of outcome measures}

The main outcome was the measure of association between eMRI and LOD whether it was reported as odds 


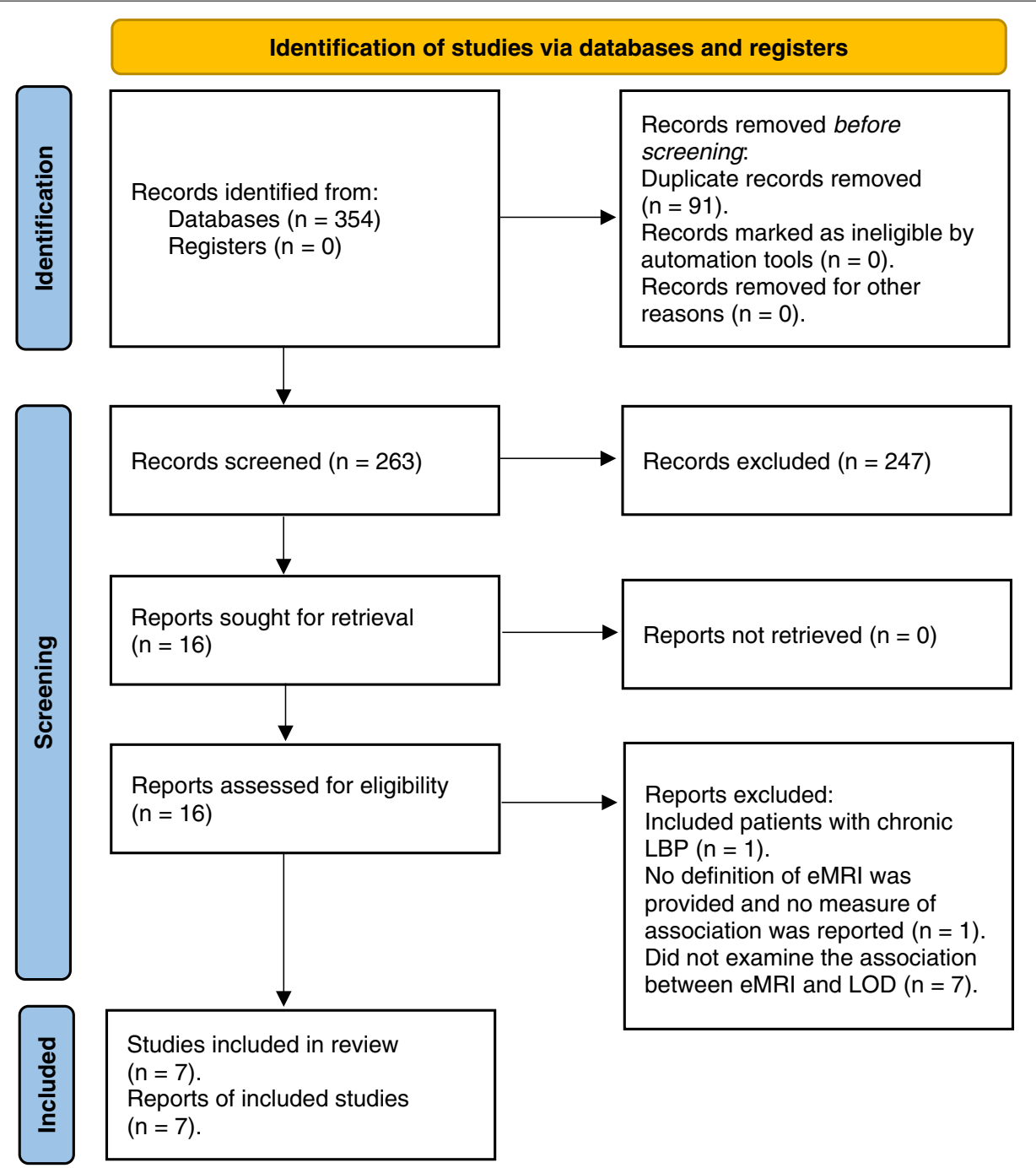

Fig. 1 The PRISMA flow diagram of studies in the review

ratios, relative risk, or mean difference in LOD between the eMRI group and the no eMRI group. The LOD was defined as the number of disability days (absence from work) due to the current episode of LBP [7, 11-13].

\section{Study selection process}

All retrieved records were imported to Covidence webbased application and duplicate records were removed. Initially, titles and abstracts of all records were screened, then full text of relevant papers were reviewed for eligibility for inclusion in the review. The study selection process was conducted independently by two reviewers, and any disagreements were resolved by discussion with a third reviewer. The reasons for study exclusions made during the second stage were reported in the PRISMA flow diagram (Fig. 1).

\section{Quality assessment}

Methodological quality assessment of included studies was conducted independently by two reviewers using the Newcastle-Ottawa scale for cohort studies (Supplemental file 3) [27] and any disagreements were resolved by discussion with a third reviewer. Where there was a conflict of interest or potential reviewer bias, the reviewer in question was not involved in the quality assessment. This tool assesses the quality of the sample selection process, comparability of cohorts, and the assessment of outcome. Each study can be given a maximum of one star for each element within the sample selection process and the outcome and a maximum of two stars can be given for the comparability section. The sample selection section evaluated the: (1) representativeness of the exposed cohort (representative of populations presenting with acute 
LBP without red flags and exposed to eMRI scanning), (2) selection of non-exposed cohort, (3) ascertainment of the exposure and (4) demonstration that the outcome was not present at the start of the study. The comparability section evaluated: (1) whether a study adjusted for the most important factors deliberately and (2) whether a study adjusted for other important risk factors. The outcome section evaluated: (1) the method used to assess the outcome, (2) whether the follow-up period was long enough for outcomes to occur and (3) loss to follow up rate. To summarize the risk of bias in each study, we converted the Newcastle-Ottawa scales to the Agency for Healthcare Research and Quality (AHRQ) standards using the following recommended thresholds [28]: (1) good quality (3 or 4 stars in selection domain and 1 or 2 stars in comparability domain and 2 or 3 stars in outcome/exposure domain); (2) fair quality (2 stars in selection domain and 1 or 2 stars in comparability domain and 2 or 3 stars in outcome/exposure domain); (3) poor quality (0 or 1 star in selection domain OR 0 stars in comparability domain OR 0 or 1 stars in outcome/exposure domain).

\section{Data extraction}

The following data were extracted: study aim, source of funding, source of data, methods of data collection, study design, setting, follow up duration, population, number of participants, demographics, definition of LBP, definition of eMRI, definition of LOD, outcomes of association between eMRI and LOD, strengths and limitations, and conclusion. Data extraction was undertaken independently by two reviewers. Any disagreements were resolved by unanimity after involving a third reviewer. Where there was a conflict of interest or potential reviewer bias, the reviewer in question was not involved in the data extraction. Contacting authors for any missing data was considered. However, all required data was presented in the included papers.

\section{Data analysis}

Meta-analysis was considered but owing to betweenstudy heterogeneity in measures of association between eMRI and LOD reported in included studies, formal pooling of the results was not feasible. Therefore, a narrative synthesis of results was conducted. Narrative synthesis was presented as reported in the original study and no additional analysis/synthesis were conducted.

\section{Results}

Study selection

Search strategies identified 354 records (Medline 93, EMBASE 187, CINAHL 74). After the removal of duplicates, 262 reports remained for the title and abstract screening. A total of 248 reports were excluded based on title and abstract. After the full-text screening, a further 9 studies did not meet the review inclusion criteria and were excluded (Fig. 1) [10, 29-36]. Three reports (Shraim et al., 2015, 2017, and 2019) $[6,7,13]$ used the same sample at the same time in the same settings but addressing different objectives. Therefore, a total of 7 studies were included in the quality assessment stage of this systematic review (Fig. 1) $[6,7,10-13,37]$.

\section{Study characteristics}

The characteristics of the included studies are presented in Table 1. All included studies were conducted in the United States (US) and used workers' compensation (WC) administrative databases. Six studies used a retrospective cohort study design [6, 7, 11-13, 37], and one study used a prospective cohort study design [10]. Three studies by Shraim and colleagues [6, 7, 13] used the same sample to examine the relationship between different individual-level variables (including eMRI) with neighborhood and state-level variables and LOD in LBP cases (see Table 3 for list of variables included in each study). The sample size ranged from 98 to 59,360 with a total number of 64,232 LBP cases in all studies. The proportions of males ranged from 69 to $73 \%$. The mean age of participants ranged between 39.4 and 41.4 years $[6,7,11,13,37]$. The median age was 34 years in one study [12], and one study included individuals aged $16-61$ years but no summary measure of age was provided [10]. All studies included cases with uncomplicated LBP identified using ICD-9 codes $[6,7,11,13,37]$, nature of injury codes [10], or combinations of body part and nature of injury codes [11]. eMRI was defined as lumbar MRI within 30 days [6, $7,11,13,37$ ] or 6 weeks of seeking medical care [10]. The LOD was defined as the total number of days of continuous paid indemnity (lost wage replacement for temporary total or temporary partial lost days) and truncated at either 1-year $[6,7,11,13,37]$ or 2 -year of follow-up periods $[11,37]$.

\section{Quality assessment}

None of the studies examined in the quality assessment stage were excluded. All included studies were of good methodological quality. Six studies scored nine stars $[6,7,11-13,37]$ and one scored eight stars due to $30 \%$ loss to follow up [10] (Table 2). The score given to the representativeness of the exposed cohort was based on the study population which may differ in 


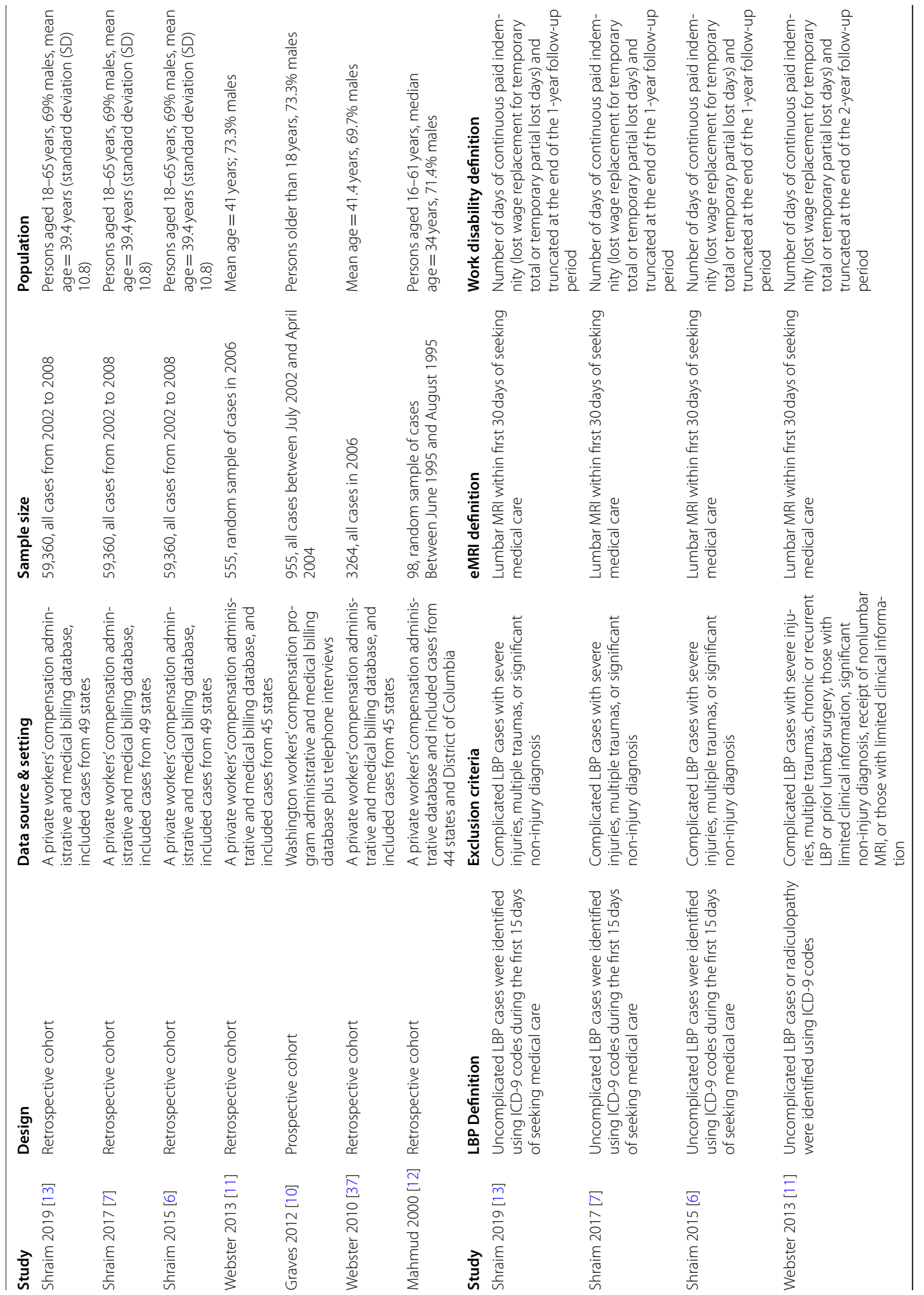




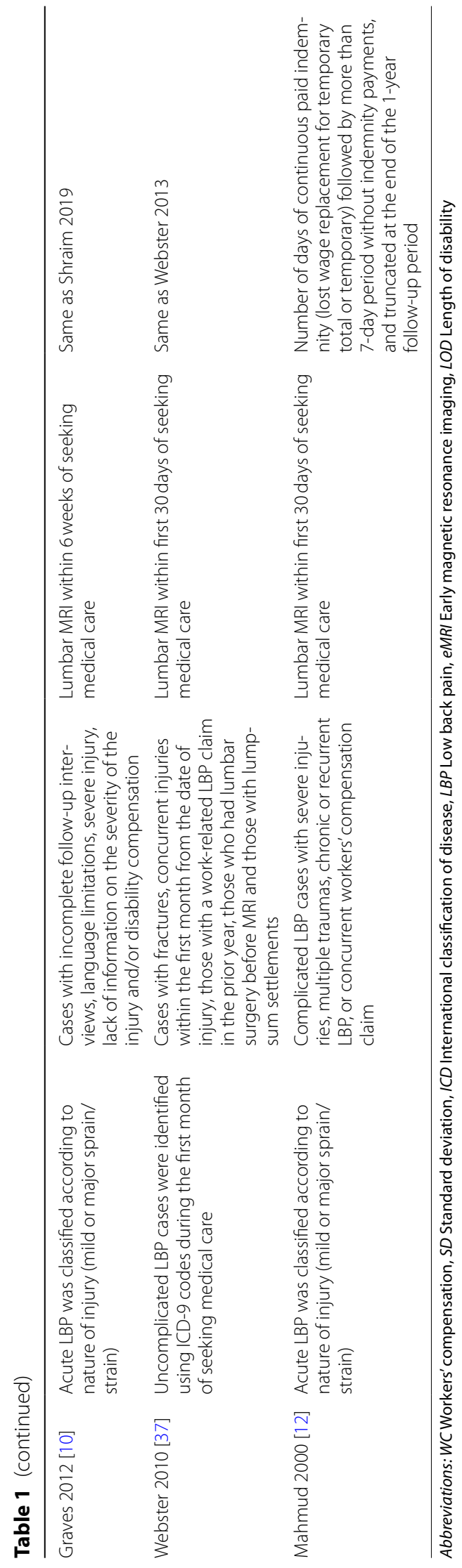


Table 2 Quality assessment of studies using the NewcastleOttawa scale

\begin{tabular}{lllll}
\hline Study & Selection & Comparability & Outcome & $\begin{array}{l}\text { Total } \\
\text { Quality } \\
\text { Score }\end{array}$ \\
\hline Shraim 2019 [13] & $* * * *$ & $* *$ & $* * *$ & 9 \\
Shraim 2017 [7] & $* * * *$ & $* *$ & $* * *$ & 9 \\
Shraim 2015 [6] & $* * * *$ & $* *$ & $* * *$ & 9 \\
Webster 2013 [11] & $* * * *$ & $* *$ & $* * *$ & 9 \\
Graves 2012 [10] & $* * * *$ & $* *$ & $* *$ & 8 \\
Webster 2010 [37] & $* * * *$ & $* *$ & $* * *$ & 9 \\
Mahmud 2000 [12] & $* * * *$ & $* *$ & $* * *$ & 9 \\
\hline
\end{tabular}

characteristics of the general population. One of the current review authors (MS) is an author in three of the included studies, therefore, MS was not involved in the quality assessment and any subsequent data extraction of Shraim and colleagues' studies [6, 7, 13]. A total of 2 out of the 7 included studies had reviewer disagreement in relation to the outcome score of Newcastle-Ottawa scale. This disagreement was resolved by referring the two studies in question to a third reviewer (BA).

\section{The association between eMRI and LOD}

All included studies investigated the association between eMRI and LOD. The studies used multivariable analyses and adjusted for potential confounders. The main variables that all studies consistently adjusted for were age and gender (Table 3). Five studies followed up the patients for a duration of 1 year [6, 7, 10, 12, 13], and reported unadjusted mean (standard deviation (SD)) of LOD of 142.2 (125.0), 142.2 (125.0), 163.5 (144.6), 115 (not reported), and 142.2 (125.0) days in the eMRI group compared to 79.6 (105.1), 79.6 (105.1), 42.6 (86.6), 13 (not reported), and 79.6 (105.1) days in the no eMRI group, respectively. One of these studies did not report the SD for the LOD [12]. Two studies [11, 37] followed up patients for a duration of 2 years and reported unadjusted means of LOD of 128.5 (95\% CI 128.5, 201.5) and 133.6 (95\% CI 120.0,146.7) days in the eMRI groups compared to $44.4(95 \%$ CI $37.5,51.4)$ and 22.9 (95\% CI 19.5, 26.2) days in the no eMRI groups, respectively. Two studies reported unadjusted means of LOD for LBP patients with radiculopathy of 184.0 (95\% CI $154.8,213.2)$ and $215.3(\mathrm{SD}=127.5)$ days in the eMRI group compared to 50.0 (95\% CI 38.0, 61.9) and 121.3 $(\mathrm{SD}=142.6)$ days in the none-eMRI group, respectively (see Table 3) [10, 11]. Three studies reported adjusted geometric mean of LOD of 39.6 (95\% CI 36.0, 43.6), 37.7 (95\% CI 33.2, 42.2), and 37.8 (95\% CI 33.9, 41.9) days in the eMRI groups compared to 25.9 (95\% CI 23.0, 29.1), 24.4 (95\% CI 21.4, 28.0), and 28.4 (95\% CI 25.4, 31.7) days in the no eMRI groups at 1-year follow up, respectively $[6,7,13]$. These three studies reported that the eMRI groups had a higher adjusted mean LOD than the no eMRI groups by 9.4 days $(95 \%$ CI $8.5,10.2)$ [13], 13.3 days ( $95 \%$ CI $11.8,14.8$ ) [7], and 13.7 days (95\% CI 13.0, 14.5) [6]. Four studies reported the hazard ratio (HR) as a measure of association between eMRI and work disability. Three studies reported that the eMRI groups had a higher HR of increased LOD than the no eMRI groups by 1.75 (95\% CI 1.23, 2.50) [10], 2.91 (95\% CI 1.45, 5.84) [12], and 3.13 (95\% CI 2.33, 4.17) [11]. Two studies $[10,11]$ reported that eMRI groups with LBP and radiculopathy had a higher HR of increased LOD than the no eMRI groups with LBP and radiculopathy by 2.08 $(95 \%$ CI 1.67, 2.63) [10] and 3.57 (95\% CI 2.33, 5.56) [11]. One study controlled for potential MRI indication bias using the propensity of belonging to the eMRI group, computed based on demographic and severity indicators with adjustment for potential residual confounding of covariates [37]. This study reported that low-propensity eMRI subgroup had a higher HR of increased LOD than the low-propensity no eMRI subgroup and high-propensity no eMRI subgroup by $3.0(95 \%$ CI $2.6,3.4)$ and 2.9 (95\% CI 2.3, 3.5), respectively [37].

\section{Discussion}

This systematic review examined the relationship between eMRI for LBP without red flags and LOD. All included studies showed that subjects who received eMRI for LBP had an increased LOD than those who did not receive eMRI. The findings of our systematic review are consistent with the findings of a previous systematic review of two studies which concluded that patients with acute non-specific LBP who received MRI had a higher LOD as compared to the no MRI group [25]. The current systematic review included 7 studies from 5 unique study populations and added further evidence that eMRI is associated with increased LOD in patients with LBP without red flags even after accounting for several factors associated with LOD in this population. The three studies by Shraim and colleagues used the same study population and found that eMRI was associated with increased LOD in patients presenting with acute LBP without red flags after accounting for neighborhood socio-economic characteristics and state-level variables, including WC policy characteristics $[6,7,13]$. One study by Graves and colleagues also showed that eMRI was associated with increased LOD in patients with LBP without red flags after accounting for baseline functional disability, pain severity, quality of life, catastrophizing, work-fear avoidance, job accommodation, previous LBP status, job satisfaction, industry, physical demands at work, and type of first medical visit [10]. 


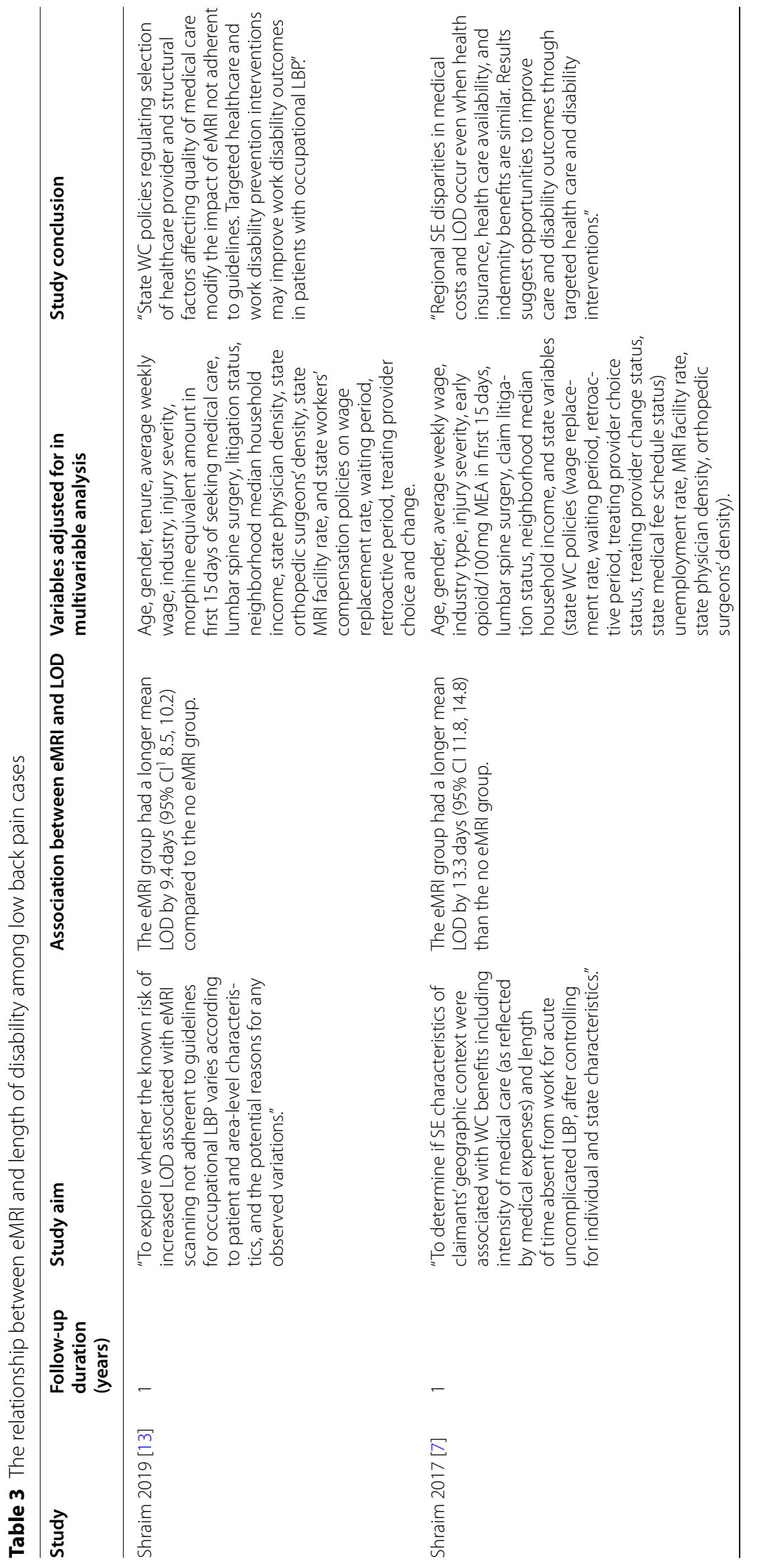




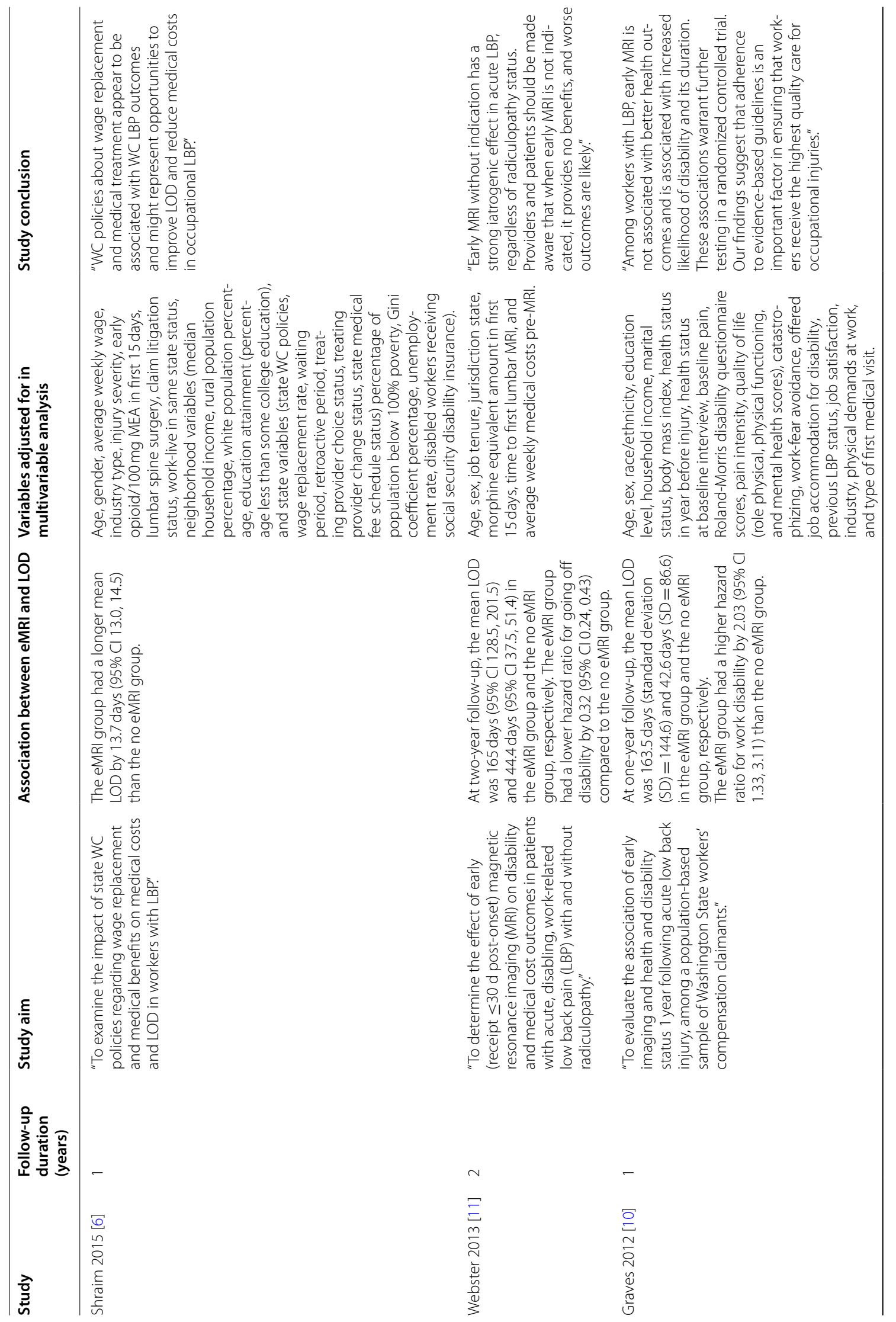




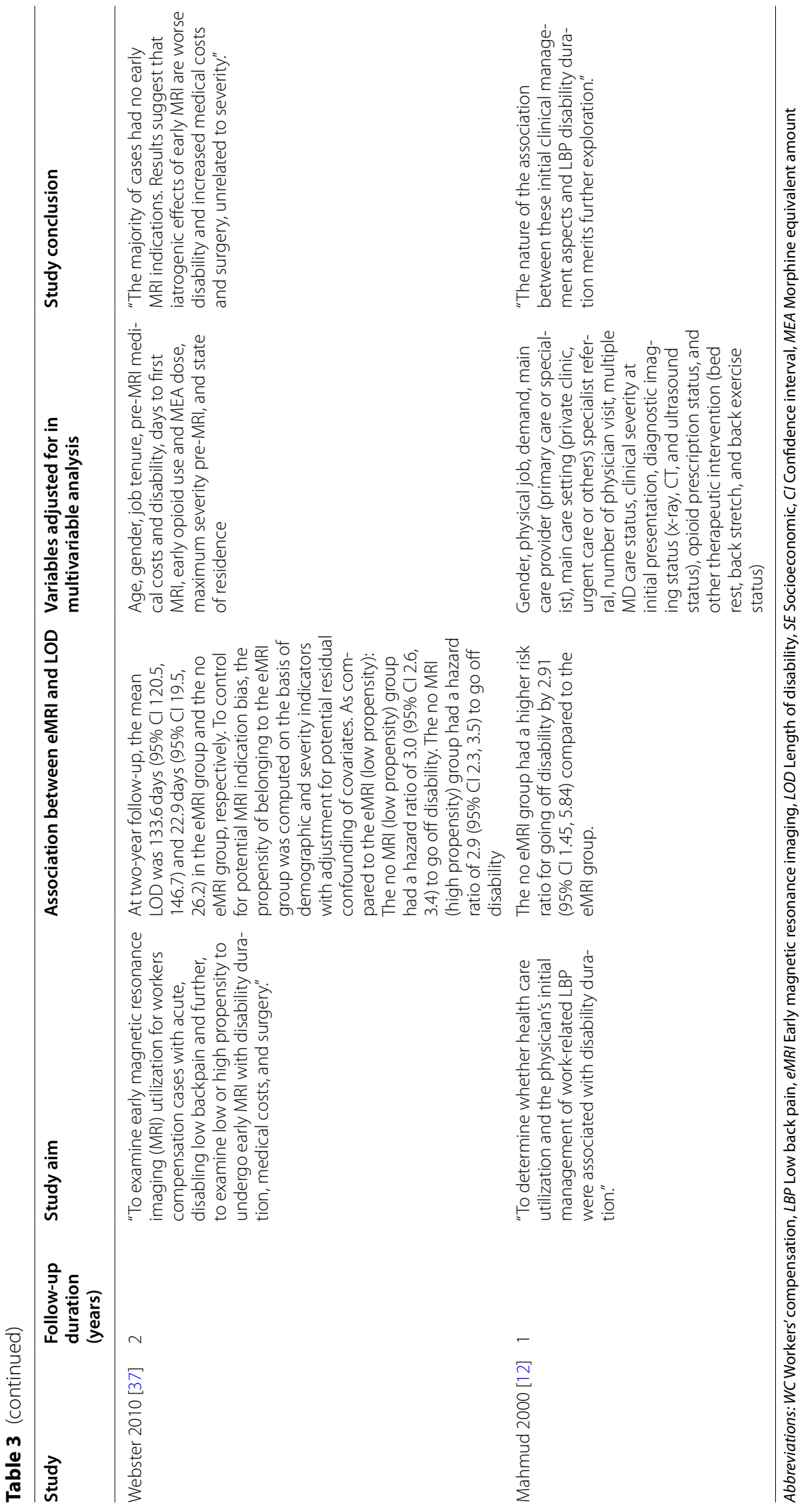


Despite recommendations of clinical practice guidelines against eMRI scanning for acute LBP without red flags, significant proportions of patient with LBP receive eMRI [10-13, 37]. The exact reasons for this are not clear. Previous studies hypothesized that lack of adherence to clinical guidelines could be explained by several factors, including patient's demand for diagnostic imaging, patient reassurance by diagnostic findings, concerns about litigation especially in WC settings, physicians' inadequate awareness about the natural history of acute LBP, and inertia of previous experience, or outcome expectancy [11,37-39].

This review used a comprehensive search strategy and searched key bibliographical databases and the grey literature to identify relevant studies. The included studies consisted of large samples of LBP cases and used WC administrative data which captures complete information on medical bills, treatment, interventions, and duration of work disability.

This review has some limitations that should be noted. First, the current review included a small number of studies (7 studies from 5 study populations). Second, the included studies in this review used WC databases as the primary source of data. This data does not provide information on some predictors of LOD, such as level of functional disability, work accommodation, nature of job, fear-avoidance, and other comorbidities, including psychiatric conditions. However, this is unlikely to influence the findings unless the distribution of those predictors differs significantly between the eMRI and no MRI groups. In addition, the study by Graves et al., found that eMRI group had an increased HR of LOD than the eMRI group even after controlling for baseline pain, Roland-Morris disability questionnaire scores, pain intensity, quality of life (role physical, physical functioning, and mental health scores), catastrophizing, work-fear avoidance, offered job accommodation for disability, previous LBP status, job satisfaction, industry, physical demands at work, and type of first medical visit [10]. Third, the included studies measured LOD using wage replacement data. This may underestimate the observed association between eMRI and increased LOD because termination of wage replacement does not necessarily translate to complete recovery or return to work. Fourth, all included studies were conducted in the US, which may limit the generalizability of the findings to other countries that have different healthcare systems. However, these studies have good methodological quality and reported consistent findings related to the review question. Fifth, formal pooling of the results using meta-analysis was not feasible owing to between-study heterogeneity.
More research is needed to uncover the exact reasons for ordering the non-indicated eMRI for acute LBP without red flags. This information is useful for developing interventions and strategies to improve adherence to clinical guidelines' recommendations about the management of patients presenting with acute LBP.

\section{Conclusions}

eMRI is associated with an increased LOD in patients with acute LBP without red flags. Further research is needed to fully understand the reasons for the use of non-indicated eMRI for patients presenting with LBP. Developing healthcare interventions to enhance adherence to clinical guidelines may improve disability outcomes among patients with LBP.

\section{Supplementary Information}

The online version contains supplementary material available at https://doi. org/10.1186/s12891-021-04863-9.

Additional file 1. Prisma 2020 Checklist.

Additional file 2. Full search strategy.

Additional file 3:. Newcastle-Ottawa quality assessment scale for cohort studies.

\section{Acknowledgements}

Not applicable.

\section{Authors' contributions}

Conceptualization, MS.; Data curation, MAS, ARI, MEE, BAS, and MS.; Formal analysis, MAS, ARI, MEE, BAS, BA, and MS.; Investigation, MS.; Methodology, MS.; Supervision, MS.; Writing - original draft, MAS, ARI, MEE, and BAS.; Writing - review \& editing, MAS, ARI, MEE, BAS, BA, and MS. All authors have read and agreed to the published version of the manuscript.

\section{Funding}

Not applicable.

Availability of data and materials

No new data were created or analyzed in this study. Data sharing is not applicable to this article.

\section{Declarations}

Ethics approval and consent to participate

Not applicable.

\section{Consent for publication}

Not applicable.

\section{Competing interests}

The authors declare no conflict of interest.

\section{Author details}

${ }^{1}$ College of Medicine, QU Health, Qatar University, Doha, Qatar. ${ }^{2} \mathrm{NHMRC}$

Centre of Clinical Research Excellence in Spinal Pain, Injury \& Health, School of Health \& Rehabilitation Sciences, The University of Queensland, Brisbane, QLD 4072, Australia. ${ }^{3}$ College of Medicine and Health Sciences, Khalifa University, Abu Dhabi, United Arab Emirates. ${ }^{4} \mathrm{KU}$ Research and Data Intelligence Support Center (RDISC) AW 8474000331, Khalifa University of Science 
and Technology, Abu Dhabi, United Arab Emirates. ${ }^{5}$ Department of Public Health, College of Health Sciences, Qatar University, QU Health, Doha, Qatar.

\section{Received: 23 July 2021 Accepted: 8 November 2021}

Published online: 24 November 2021

\section{References}

1. Wu A, March L, Zheng X, Huang J, Wang X, Zhao J, et al. Global low back pain prevalence and years lived with disability from 1990 to 2017: estimates from the global burden of disease study 2017. Ann Transl Med. 2020;8(6):299.

2. Katz JN. Lumbar disc disorders and low-back pain: socioeconomic factors and consequences. J Bone Joint Surg Am. 2006;88(Suppl 2):21-4.

3. Xie Y, Bowe B, Mokdad AH, Xian H, Yan Y, Li T, et al. Analysis of the global burden of disease study highlights the global, regional, and national trends of chronic kidney disease epidemiology from 1990 to 2016. Kidney Int. 2018;94(3):567-81.

4. Steenstra IA, Verbeek JH, Heymans MW, Bongers PM. Prognostic factors for duration of sick leave in patients sick listed with acute low back pain: a systematic review of the literature. Occup Environ Med. 2005;62(12):851-60.

5. Shaw WS, Pransky G, Fitzgerald TE. Early prognosis for low back disability: intervention strategies for health care providers. Disabil Rehabil. 2001;23(18):815-28.

6. Shraim M, Cifuentes M, Willetts $J$, Marucci-Wellman HR, Pransky G. Length of disability and medical costs in low back pain: do state workers' compensation policies make a difference? J Occup Environ Med. 2015;57(12):1275-83.

7. Shraim M, Cifuentes M, Willetts JL, Marucci-Wellman HR, Pransky G. Regional socioeconomic disparities in outcomes for workers with low back pain in the United States. Am J Ind Med. 2017;60(5):472-83.

8. Webster BS, Verma SK, Gatchel RJ. Relationship between early opioid prescribing for acute occupational low back pain and disability duration, medical costs, subsequent surgery and late opioid use. Spine (Phila Pa 1976). 2007;32(19):2127-32.

9. Webster BS, Choi Y, Bauer AZ, Cifuentes M, Pransky G. The cascade of medical services and associated longitudinal costs due to nonadherent magnetic resonance imaging for low back pain. Spine (Phila Pa 1976). 2014;39(17):1433-40.

10. Graves JM, Fulton-Kehoe D, Jarvik JG, Franklin GM. Early imaging for acute low back pain: one-year health and disability outcomes among Washington state workers. Spine (Phila Pa 1976). 2012;37(18):1617-27.

11. Webster BS, Bauer AZ, Choi Y, Cifuentes M, Pransky GS. latrogenic consequences of early magnetic resonance imaging in acute, work-related, disabling low back pain. Spine (Phila Pa 1976). 2013;38(22):1939-46.

12. Mahmud MA, Webster BS, Courtney TK, Matz S, Tacci JA, Christiani DC Clinical management and the duration of disability for work-related low back pain. J Occup Environ Med. 2000;42(12):1178-87.

13. Shraim M, Cifuentes $M$, Willetts JL, Marucci-Wellman HR, Pransky G. Why does the adverse effect of inappropriate MRI for LBP vary by geographic location? An exploratory analysis. BMC Musculoskelet Disord. 2019;20(1):574.

14. Boos N, Semmer N, Elfering A, Schade V, Gal I, Zanetti M, et al. Natural history of individuals with asymptomatic disc abnormalities in magnetic resonance imaging: predictors of low back pain-related medical consultation and work incapacity. Spine (Phila Pa 1976). 2000;25(12):1484-92.

15. Jarvik JJ, Hollingworth W, Heagerty P, Haynor DR, Deyo RA. The longitudinal assessment of imaging and disability of the Back (LAIDBack) study: baseline data. Spine (Phila Pa 1976). 2001;26(10):1158-66.

16. Babińska A, Wawrzynek W, Czech E, Skupiński J, Szczygieł J, Łabuz-Roszak B. No association between MRI changes in the lumbar spine and intensity of pain, quality of life, depressive and anxiety symptoms in patients with low back pain. Neurol Neurochir Pol. 2019;53(1):74-82.

17. Chou R, Fu R, Carrino JA, Deyo RA. Imaging strategies for low-back pain: systematic review and meta-analysis. Lancet. 2009;373(9662):463-72.

18. Chou R, Qaseem A, Snow V, Casey D, Cross JT Jr, Shekelle P, et al. Diagnosis and treatment of low back pain: a joint clinical practice guideline from the American College of Physicians and the American pain society. Ann Intern Med. 2007;147(7):478-91.
19. Bernstein IA, Malik Q, Carville S, Ward S. Low back pain and sciatica: summary of NICE guidance. BMJ. 2017;356:16748.

20. Patel ND, Broderick DF, Burns J, Deshmukh TK, Fries IB, Harvey HB, et al. ACR appropriateness criteria low back pain. J Am Coll Radiol. 2016;13(9):1069-78.

21. Stochkendahl MJ, Kjaer P, Hartvigsen J, Kongsted A, Aaboe J, Andersen M, et al. National Clinical Guidelines for non-surgical treatment of patients with recent onset low back pain or lumbar radiculopathy. Eur Spine J. 2018;27(1):60-75

22. Jenkins HJ, Downie AS, Maher CG, Moloney NA, Magnussen JS, Hancock MJ. Imaging for low back pain: is clinical use consistent with guidelines? A systematic review and meta-analysis. Spine J. 2018;18(12):2266-77.

23. Lurie JD, Birkmeyer NJ, Weinstein JN. Rates of advanced spinal imaging and spine surgery. Spine (Phila Pa 1976). 2003;28(6):616-20.

24. Jarvik JG, Hollingworth W, Martin B, Emerson SS, Gray DT, Overman S, et al. Rapid magnetic resonance imaging vs radiographs for patients with low back pain: a randomized controlled trial. JAMA. 2003;289(21):2810-8.

25. Lemmers GPG, van Lankveld W, Westert GP, van der Wees PJ, Staal JB. Imaging versus no imaging for low back pain: a systematic review, measuring costs, healthcare utilization and absence from work. Eur Spine J. 2019:28(5):937-50.

26. Moher D, Liberati A, Tetzlaff J, Altman DG. Preferred reporting items for systematic reviews and meta-analyses: the PRISMA statement. Plos Med. 2009;6(7):e1000097

27. Wells G, Shea B, DL OC, Peterson J, Welch V, Losos M, et al. The NewcastleOttawa scale (NOS) for assessing the quality of nonrandomised studies in meta-analyses, vol. 2014; 2014

28. Viswanathan M, Ansari MT, Berkman ND, Chang S, Hartling L, McPheeters LM, et al. Assessing the Risk of Bias of Individual Studies in Systematic Reviews of Health Care Interventions. In: Agency for Healthcare Research and Quality Methods Guide for Comparative Effectiveness Reviews; 2012.

29. Kummel BM. Nonorganic signs of significance in low back pain. Spine (Phila Pa 1976). 1996;21(9):1077-81.

30. Carragee EJ, Alamin TF, Miller JL, Carragee JM. Discographic, MRI and psychosocial determinants of low back pain disability and remission: a prospective study in subjects with benign persistent back pain. Spine J. 2005:5(1):24-35.

31. Kovacs FM, Fernández C, Cordero A, Muriel A, González-Luján L, Gil del Real MT. Non-specific low back pain in primary care in the Spanish National Health Service: a prospective study on clinical outcomes and determinants of management. BMC Health Serv Res. 2006;6:57.

32. Bouton $C$, Roche $G$, Roquelaure $Y$, Legrand $E$, Penneau-Fontbonne D, Dubus $V$, et al. Management of low back pain in primary care prior to multidisciplinary functional restoration: a retrospective study of 72 patients. Ann Readapt Med Phys. 2008;51(8):650-656-6-662.

33. Jensen OK, Stengaard-Pedersen K, Jensen C, Nielsen CV. Prediction mode for unsuccessful return to work after hospital-based intervention in low back pain patients. BMC Musculoskelet Disord. 2013;14:140.

34. Jensen OK, Nielsen CV, Stengaard-Pedersen K. One-year prognosis in sicklisted low back pain patients with and without radiculopathy. Prognostic factors influencing pain and disability. Spine J. 2010;10(8):659-75.

35. Graves JM, Fulton-Kehoe D, Jarvik JG, Franklin GM. Impact of an advanced imaging utilization review program on downstream health care utilization and costs for low back pain. Med Care. 2018:56(6):520-8.

36. Savych B, Neumark D, Lea R. Do opioids help injured workers recover and get back to work? The impact of opioid prescriptions on duration of temporary disability. Ind Relat. 2019;58(4):549-90.

37. Webster BS, Cifuentes M. Relationship of early magnetic resonance imaging for work-related acute low back pain with disability and medical utilization outcomes. J Occup Environ Med. 2010;52(9):900-7.

38. Cabana MD, Rand CS, Powe NR, Wu AW, Wilson MH, Abboud PA, et al. Why don't physicians follow clinical practice guidelines? A framework for improvement. JAMA. 1999:282(15):1458-65.

39. Little P, Dorward M, Warner G, Stephens K, Senior J, Moore M. Importance of patient pressure and perceived pressure and perceived medical need for investigations, referral, and prescribing in primary care: nested observational study. BMJ. 2004:328(7437):444

\section{Publisher's Note}

Springer Nature remains neutral with regard to jurisdictional claims in published maps and institutional affiliations. 